Revue Revue de l'histoire des religions

de Ihistoire des religions

Un dieu peut-il mourir ? Enquête sur la fin des cultes dans l'Antiquité gréco-romaine

\title{
La « barbarisation » de Poséidonia et la fin des cultes grecs à Paestum
}

The "barbarisation" of Poseidonia and the end of Greek cults in Paestum

Michel Humm

\section{OpenEdition}

\section{Journals}

Édition électronique

URL : http://journals.openedition.org/rhr/8913

DOI : $10.4000 /$ rhr.8913

ISSN : 2105-2573

Éditeur

Armand Colin

Édition imprimée

Date de publication : 1 juin 2018

Pagination : 353-372

ISBN : 978-2-200-93177-3

ISSN : 0035-1423

Référence électronique

Michel Humm, «La «barbarisation » de Poséidonia et la fin des cultes grecs à Paestum », Revue de

I'histoire des religions [En ligne], 2 | 2018, mis en ligne le 01 juin 2020, consulté le 15 janvier 2021. URL http://journals.openedition.org/rhr/8913 ; DOI : https://doi.org/10.4000/rhr.8913 


\section{La « barbarisation » de Poséidonia et la fin des cultes grecs à Paestum}

Lefr. 124 Wehrli d'Aristoxène surla « barbarisation » des Poséidoniates et la fin des cultes grecs à Poséidonia renvoie à l'attraction politique et culturelle que Rome exerçait sur une partie des élites aristocratiques lucaniennes au cours de la seconde moitié du IV siècle av. J.-C., particulièrement vers la fin du siècle. Ce processus culturel d'"autoromanisation " fut favorisé par l'avancée des Romains en Campanie et en Italie du Sud et s'inscrivait dans la durée et le temps long, mais il se conclut brutalement en 273 av. J.-C. par la déduction de la colonie de Paestum et la fondation sur place d'une nouvelle cité. Les dieux de la cité grecque de Poséidonia étaient alors définitivement morts et avaient été remplacés par les dieux romains de la colonie de Paestum.

\section{The "barbarisation" of Poseidonia and the end of Greek cults in Paestum}

Fr. 124 Wehrli of Aristoxenus, on the "barbarisation" of the Poseidonians and the end of Greek cults in Poseidonia, refers to the political and cultural attraction Rome held for a section of the Lucanian aristocratic elites during the second half of the $4^{\text {th }}$ century $B C$, particularly towards the end of the century. This cultural process of "self-Romanisation" was furthered by the Romans' advance into Campania and southern Italy and developed gradually over a long period of time; but it ended abruptly in 273 BC with the deduction of the colony of Paestum and the founding of a new city on the spot. The gods of the Greek city of Poseidonia were by then truly dead and had been replaced by the Roman gods of the colony of Paestum. 
Dans la plupart des cités du monde antique, qu'elles fussent grecques, italiques ou romaines, la religion publique était liée à l'idéal de la cité et à l'existence de la communauté civique (elle ne concernait l'individu qu'en tant que membre de la communauté des citoyens). Le culte public était constitué par un ensemble d'obligations et de rites destinés à assurer la bienveillance et la protection des dieux de la cité envers la communauté civique : la religion était donc de nature essentiellement «sociale» et "politique», et permettait de définir l'identité de chaque communauté civique'. Dans ce contexte, la disparition d'une cité ou sa transformation en une nouvelle communauté civique pouvait poser la question de la fin des cultes. C'est ce processus qui semble à l'œuvre dans les cités grecques d'Italie du Sud, au tournant des IV $^{\mathrm{e}}$ et $\mathrm{III}^{\mathrm{e}}$ siècles, lorsque celles-ci furent touchées par la progressive «romanisation» de la région et son passage sous l'autorité de Rome. C'est particulièrement le cas pour la cité de Poséidonia-Paestum, ballotée entre fondations, refondations, déductions et passages sous diverses influences politiques et culturelles.

Dans un fragment maintes fois étudié et commenté, Aristoxène de Tarente déplore la «barbarisation» de Poséidonia du fait des Étrusques ou des Romains : celle-ci se serait traduite par la disparition de la langue et des usages grecs, ainsi que par la fin des cultes grecs traditionnels dans la cité2. L'intention nécessairement polémique de l'affirmation d'Aristoxène, un philosophe grec qui vécut dans la seconde moitié du IV $V^{\mathrm{e}}$ et au début du III ${ }^{\mathrm{e}}$ siècle, doit être replacée dans le contexte des bouleversements géopolitiques et culturels qui affectèrent la région à cette époque, et peut être confrontée aux données fournies par l'archéologie, qui témoignent des transformations socio-culturelles et socio-politiques qui affectèrent l'ancienne cité grecque, puis grécolucanienne, jusqu'à sa transformation en une cité « romaine » après la déduction de la colonie de Paestum en 273 av. J.-C.

1. John Scheid, Religion et piété à Rome [1985], Paris, Albin Michel, 2001, en particulier p. 24-27; p. 30-34; p. 47-76; Id., Les dieux, l'État et l'individu. Réflexions sur la religion civique à Rome, Paris, Seuil, 2013, p. 65-120 ; p. 165-174 ; Id., La religion des Romains [2002], Paris, Armand Colin, 2017, p. 25-29.

2. Aristoxène, fr. 124 Wehrli = Fragmenta Historicorum Graecorum II, 291 (cité infra n. 3). 
On peut dès lors se demander s'il existe un rapport, direct ou indirect, entre le témoignage d'une source littéraire dont la datation est par ailleurs problématique, et celui de l'archéologie sur les transformations urbanistiques et culturelles (mais aussi cultuelles) que connut la cité à partir de 273 av. J.-C. De quelle nature a pu être la «barbarisation» évoquée par Aristoxène et quel lien a-t-elle pu avoir avec la fin des cultes grecs à Poséidonia ? Quels sont les éléments de continuité ou de rupture entre les phénomènes observés par le philosophe à la fin du $\mathrm{IV}^{\mathrm{e}}$ siècle et la déduction par Rome de la colonie de Paestum en 273 av. J.-C. ?

\section{Le témoignage d'Aristoxène de TARENTE : Les habitants de Poséidonia Se barbarisent EN DEVENANT DES ÉTRUSQUES OU DES ROMAINS}

En conclusion d'une longue réflexion sur les mutations qu'a connues la pratique musicale au cours des époques successives, Athénée, au livre XIV des Deipnosophistes, cite un long fragment d'Aristoxène dans lequel celui-ci pousse une complainte sur la décadence de la musique de son époque par rapport à celle qui a existé par le passé, et donne à ce propos l'exemple de Poséidonia :

C'est pourquoi Aristoxène écrit dans les Symmikta sympotika : « Nous nous comportons, dit-il, comme les Poséidoniates qui habitent dans le golfe "tyrsénien". Ceux-ci, qui à l'origine étaient des Grecs, se sont barbarisés en devenant des Étrusques ou des Romains ; ils ont changé de langue ainsi que le reste de leurs coutumes, mais célèbrent aujourd'hui encore une seule de leurs fêtes grecques, à l'occasion de laquelle ils se réunissent pour rappeler le souvenir des anciens mots et des anciennes institutions tout en se lamentant auprès des uns et des autres, puis, après avoir répandu beaucoup de larmes, s'en retournent chez eux. Ainsi donc, dit Aristoxène, nous aussi, après que les théâtres se sont barbarisés et que cette musique si répandue s'est grandement corrompue, désormais peu nombreux, nous nous rappelons entre nous ce qu'était la musique. » Telles sont les paroles d'Aristoxène ${ }^{3}$.

Aristoxène, fr. 124 Wehrli $=F H G$ II, 291

(= Athénée, Deipnosophistes, xIv, 31 [632 a])

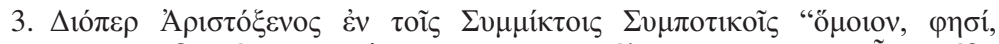

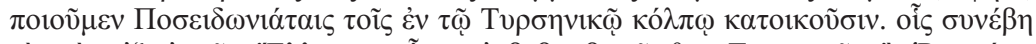

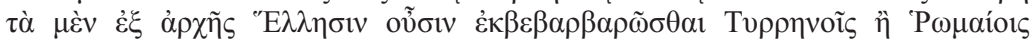


Athénée semble citer textuellement le passage d'une œuvre perdue d'Aristoxène, les Symmikta sympotika (Mélanges de propos de festins). Dans ce fragment, l'évolution culturelle de Poséidonia sert à illustrer ce qu'Aristoxène considère comme une "décadence» de la qualité musicale et théâtrale : à l'image de ce qui se passe dans la cité poséidoniate, où l'atellane d'origine campanienne a remplacé l'art du théâtre grec classique, et où la langue grecque tend à disparaître jusque dans les cultes dont ne subsisterait plus qu'une unique fête

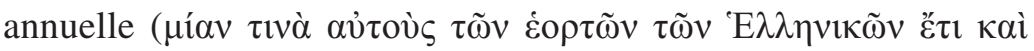
vṽv), la musique contemporaine est désormais marquée par les rythmes et les instruments de la «musique nouvelle ${ }^{4}$. L'exemple de Poséidonia n'est qu'un prétexte pour dénoncer la «décadence » de la musique et du théâtre contemporains en Grande Grèce, où les influences étrangères «barbares » sont clairement accusées d'être responsables de cette évolution. Le débat est donc placé au niveau culturel, et non au niveau politique : il n'est par conséquent pas nécessaire de rapprocher ce fragment d'Aristoxène de l'évolution politique qu'a connue Poséidonia au moment de la déduction de la colonie de Paestum, en 273 av. J.-C. Ce rapprochement est d'autant moins nécessaire que l'œuvre d'Aristoxène peut difficilement avoir été rédigée après 273 : probablement né vers $370 / 365$ av. J.-C., il semble difficile d'en faire un témoin de la déduction par Rome de la colonie de Paestum, en 273, ou d'utiliser le fr. 124 Wehrli comme un témoignage grec des conséquences culturelles de cette

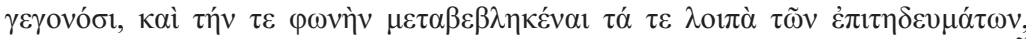

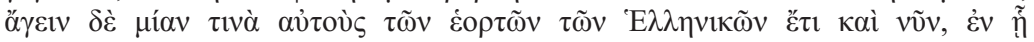

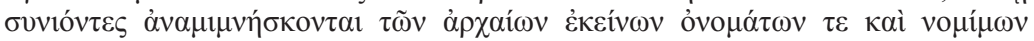

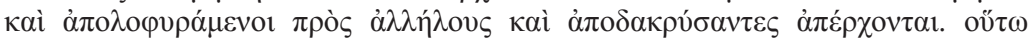

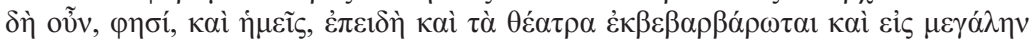

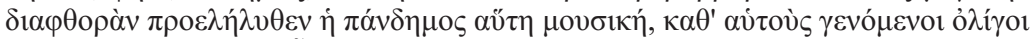

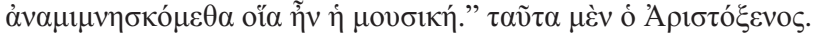

4. Voir Paola Zancani-Mantuoro, «Qualche documenti dell'arte e dell'artigianato », La monetazione di bronzo di Poseidonia-Paestum. Atti del III Convegno del Centro Internazionale di Studi Numismatici (Napoli 19-23 Aprile 1971), Rome, Istituto Italiano di Numismatica (suppl. «Annali », 18-19), 1973, p. 11-23, ici p. 16-18, sur la «barbarisation » des théâtres et les progrès de l'atellane en Italie du Sud. À propos de la musique «nouvelle » dénoncée par Aristoxène, voir Amedeo Visconti, Aristosseno di Taranto. Biografia e formazione spirituale, Naples, Centre Jean Bérard, 1999, p. 100-118 et p. 129-156. 
déduction ${ }^{5}$. Le témoignage d'Aristoxène sur Poséidonia reflète par conséquent une réalité historique qui doit se situer au cours de la seconde moitié ou vers la fin du Iv ${ }^{\mathrm{e}}$ siècle av. J.-C. ${ }^{6}$.

Selon Aristoxène, les Poséidoniates de son temps, qui avaient été des Grecs à l'origine, se seraient donc "barbarisés » ( $\dot{\kappa} \kappa \varepsilon \beta \beta \alpha \rho \beta \alpha \rho \tilde{\omega} \sigma \theta \alpha \mathrm{l})$ «en devenant des Étrusques ou des Romains »

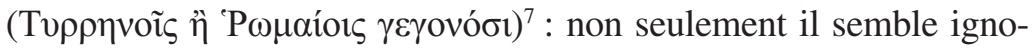
rer la présence sur place des Lucaniens, qu'il assimilait pourtant aussi à des barbares (voir infra), mais il ne fait pas de différence entre Étrusques et Romains, considérés comme interchangeables et assimilés à la même réalité ethnique et culturelle. Cette confusion délibérée entre Étrusques et Romains est assez caractéristique de certains auteurs grecs du IV siècle hostiles à l'expansion de Rome en Italie : elle avait pour but d'assimiler les Romains aux ennemis « héréditaires » des Grecs en Italie, considérés comme des barbares

5. Les informations biographiques sur Aristoxène ont été reprises et étudiées en détail par A. Visconti, Aristosseno, p. 11-20. Il n'est pas possible de suivre ceux qui, pour rendre le témoignage du fr. 124 Wehrli compatible avec une date postérieure à la déduction de la colonie de Paestum, ont proposé de rabaisser la chronologie d'Aristoxène ou de voir dans les Symmikta sympotika un ouvrage apocryphe postérieur à 273 : voir Marcello Gigante, «La cultura a Taranto », Taranto nella civiltà della Magna Grecia. Atti del X convegno di studi sulla Magna Grecia (Taranto, 4-11 ottobre 1970), Naples, Arte tipografica, 1971, p. 67-131, ici p. 75-76; Mario Torelli, «Paestum romana », Poseidonia-Paestum. Atti del XXVII convegno di studi sulla Magna Grecia (Taranto-Paestum, 9-15 ottobre 1987), Tarente, Istituto per la storia e l' archeologia della Magna Grecia, 1988, p. 33-115, ici p. 96-98 ; Id., « Roman Paestum », Tota Italia. Essays in the Cultural Formation of Roman Italy, Oxford, Clarendon Press, 1999, p. 43-88, ici p. 77-78.

6. David Ashery, «Colonizzazione e decolonizzazione », I Greci, t. I : Noi e i Greci, dir. Salvatore Settis, Turin, Einaudi, 1996, p. 73-115, ici p. 91 (dans les années 340/330) ; Fausto Zevi, «Alessandro il Molosso e Roma », Alessandro il Molosso e $i$ "condottieri » in Magna Grecia. Atti del XLIII Convegno di Studi sulla Magna Grecia (Taranto-Cosenza, 26-30 settembre 2003), Tarente, Istituto per la Storia e l'Archeologia, 2004, p. 793-832, ici p. 806-807 et n. 39.

7. Le texte du fragment transmis par Athénée ne doit pas être remis en cause, notamment en l'expurgeant de l'allusion aux Étrusques et aux Romains : voir Santo Mazzarino, Il pensiero storico classico, t. II, 1, Rome-Bari, Laterza, 1966, p. 98 ; Augusto Fraschetti, « Aristosseno, i Romani e la "barbarizzazione" di Poseidonia », Annali dell'Istituto universitario Orientale di Napoli (Archeologia e storia antica), t. 3, 1981, p. 97-115, ici p. 99 ; cf. Ulrich von Wilamowitz-Moellendorff, apparat critique dans Athenaei Naucratitae Dipnosophistarum libri XV, recensuit Georgius Kaibel, t. III : Libri XI-XV-Indices, 1890, Leipzig, Teubner, p. 394 ; Erik Wikén, Die Kunde der Hellenen von dem Lande und den Völkern der Apenninenhalbinsel bis 300 v. Chr., Lund, H. Ohlssons Buchdruckerei, 1937, p. 185, n. 3 ; Hans Riemann, s.v. «Poseidonia (Paestum)», dans Realencyclopädie der classischen Altertumswissenschaft, vol. XXII, 1, 1953, col. 1230-1254, ici col. 1232. 
par définition, et faisait partie de la propagande diplomatique de Syracuse et de Tarente contre Rome au IV siècle. En même temps,

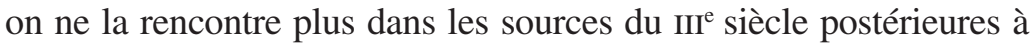
la guerre de Pyrrhus, ce qui confirme la datation du fragment et son attribution à Aristoxène ${ }^{8}$.

Le rapprochement par Aristoxène entre Étrusques et Romains est donc volontaire et délibéré, dans le but évident de présenter les Romains comme des «Barbares » : Poséidonia est d'ailleurs située

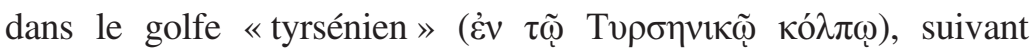

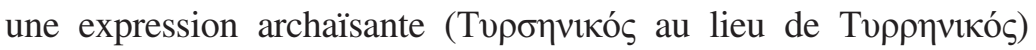
qui permet de renforcer le caractère étrusque de la Poséidonia contemporaine en évoquant l'ancienne thalassocratie étrusque en mer Tyrrhénienne et le conflit ancestral entre Grecs et (barbares) Étrusques pour son contrôle 9 . En faisant des Romains des «barbares », Aristoxène s'inscrit dans une tradition déjà présente chez Aristote, qui avait rangé les coutumes romaines dans les Nomima barbarica $^{10}$. Le témoignage d'Aristoxène peut être rapproché de celui de Strabon, qui évoque, dans des termes identiques, la «bar-

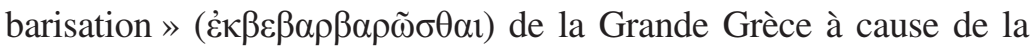
présence des Lucaniens, des Brettiens et des Campaniens, «qui sont d'ailleurs Campaniens de nom seulement et Romains de fait, puisqu'ils sont devenus citoyens romains $»^{11}$. La source de ce passage de Strabon est généralement identifiée à Posidonius, mais pourrait remonter à Timée, voire à Aristoxène : aux yeux de certains

8. A. Fraschetti, «Aristosseno », p. 104-106; Domenico Musti, «Etruschi e Greci nella rappresentazione dionisiana delle origini di Roma», Gli Etruschi e Roma. Incontro di studio in onore di Massimo Pallottino (Roma, 11-13 dicembre 1979), Rome, Giorgio Bretschneider, 1981, p. 23-44, ici p. 26-27; Gabriella Vanotti, «Roma polis Hellenis, Roma polis Tyrrhenis. Riflessioni sul tema », Mélanges de l'École française de Rome - Antiquité, t. 111, fasc. 1, 1999, p. 217-255 ; Federicomaria Muccioli, «La letteratura storiografica tra Filisto e Timeo », Storici greci d'Occidente, dir. Riccardo Vattuone, Bologne, Il Mulino, 2002, p. 137-176, ici p. 147-154.

9. Voir Dominique Briquel, Les Tyrrhènes, peuple des tours, Rome, École française de Rome («Collection de l'École française de Rome», 178), 1993, p. 196-199.

10. Aristote, fr. 604 Rose (= Varron, Langue latine, vII, 70) ; voir M. Humm, "Aristote et les Romains : entre hellénisme et barbarie, une vision grecque de Rome du $\mathrm{IV}^{\mathrm{e}}$ siècle avant J.-C. », Aristoteles Romanus. La réception de la science aristotélicienne dans l'Empire gréco-romain, dir. Yves Lehmann, Turnhout, Brepols, 2013, p. 425-462.

11. Strabon, Géographie, VI, 1, 2. 
Grecs en tout cas, la « romanisation » de la Grande Grèce eut pour conséquence sa «barbarisation $»^{12}$. Derrière le conflit «culturel» entre hellénisme et barbarie transparaît par conséquent un conflit de nature géopolitique : mais pourquoi alors avoir sciemment passé sous silence le rôle joué par les Lucaniens dans la «barbarisation » de Poséidonia, et dans quelle mesure celle-ci a-t-elle pu également affecter les cultes grecs de la cité ?

\section{«LUCANISATION » OU « ROMANISATION »?}

\section{LA « Barbarisation 》 DE Poséidonia AU Temps d'Aristoxène}

La «barbarisation » dénoncée par Aristoxène ne se limite pas à la musique ou au théâtre, mais concerne également l'usage du grec ainsi que les cultes publics, puisque les Poséidoniates auraient « changé de langue ainsi que le reste de leurs coutumes », en ne célébrant plus qu'une fois par an «une seule de leurs fêtes grecques ». Le jugement d'Aristoxène a donc une portée générale qui touche à trois des quatre critères qui définissaient, selon Hérodote, l'appartenance au monde grec, puisque celui-ci est, selon lui, « uni par la langue et par le sang, les sanctuaires et les sacrifices qui nous sont communs, et nos mœurs qui sont les mêmes $»^{13}$. Pour les Grecs, la «barbarisation » n'est pas entendue comme la conséquence d'une domination politique étrangère, mais comme la perte de l'identité culturelle hellénique ${ }^{14}$. Comme

12. Strabon, Géographie, t. III : Livres V et VI, éd. François Lasserre, Paris, Les Belles Lettres («Collection des Universités de France»), 1967, p. 220

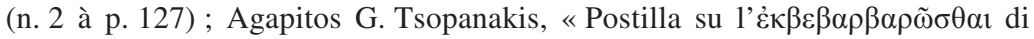
Strabone », Parola del Passato, t. 39, 1984, p. 139-143 ; D. Musti, "Sanniti, Lucani e Brettii nella Geografia di Strabone (Sulla storia del nome dei Lucani) » [1985], Strabone e la Magna Grecia. Città e popoli dell'Italia antica, Padoue,

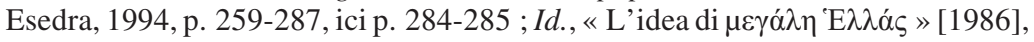
ibid., p. 61-94, ici p. 68-77 ; Glen W. Bowersock, «Les Grecs "barbarisés" », Ktèma, t. 17, 1992, p. 249-257; Mathilde Simon, Le rivage grec de l'Italie romaine. La Grande Grèce dans l'historiographie augustéenne, Rome, École française de Rome («Collection de l'École française de Rome», 442), 2011, p. 380-390.

13. Hérodote, L'Enquête, vIII, 144, trad. Denis Roussel, Paris, Gallimard, 1964. Voir F. Zevi, « Alessandro il Molosso », p. 803-804.

14. D. Asheri, «Colonizzazione e decolonizzazione », dans I Greci, p. 72-115, ici p. 93-100 ; G. W. Bowersock, "Les Grecs "barbarisés" », passim ; F. Zevi, ibid., p. 807-808. 
l'avaient déjà remarqué S. Mazzarino et $\mathrm{A}$. Fraschetti, Aristoxène ne fait pas allusion, dans ce fragment, à une occupation brutale de Poséidonia par les Romains, qui aurait modifié d'un seul coup les modes de vie et les pratiques religieuses des habitants par une intégration culturelle imposée de l'extérieur, mais il dénonce un processus de "romanisation» dont les Poséidoniates seraient eux-mêmes les acteurs et qui les fait devenir des barbares ${ }^{15}$. Mais qui étaient exactement les Poséidoniates dont parle notre auteur?

Dans sa biographie de Pythagore, en voulant montrer l'audience de l'enseignement du Maître auprès des populations barbares d'Italie, Aristoxène mentionnait les Romains après les Lucaniens, les Messapiens et les Peucètes ${ }^{16}$. On sait que, dans cet ouvrage, l'auteur transposait à l'époque du philosophe les réalités géopolitiques du $\mathrm{IV}^{\mathrm{e}}$ siècle finissant, et son énumération suit peut-être un ordre géographique d'ouest en est et du sud au nord (par rapport à Tarente), dans lequel la place des Romains s'expliquerait par leur présence à Lucérie, une colonie déduite en Apulie en 314 av. J.-C. ${ }^{17}$. Il rangeait donc les Romains parmi les autres populations barbares d'Italie, aux côtés notamment des Lucaniens, ce qui corrobore le témoignage du fr. 124 Wehrli sur la barbarisation des Poséidoniates du fait des Romains (assimilés à des Étrusques) ${ }^{18}$. Au cours du IV $\mathrm{IV}^{\mathrm{e}}$ siècle en effet, non seulement Poséidonia fut prise et occupée par les Lucaniens, une population italique d'origine sabellique (ou samnite) et de langue osque, mais certains d'entre eux se rapprochèrent des Romains ou eurent des

15. S. Mazzarino, Il pensiero, p. 98 ; A. Fraschetti, «Aristosseno», p. 111 ; M. Simon, Le rivage grec, p. 381-383.

16. Aristoxène, fr. 17 Wehrli (= Porphyre, Vie de Pythagore, 22) à propos des Lucaniens, Messapiens, Peucètes et Romains affluant auprès de Pythagore. Voir Pierre Wuilleumier, Tarente, des origines à la conquête romaine («Bibliothèque des Écoles françaises d'Athènes et de Rome», 148), Paris, De Boccard, 1939, p. 604-605, ou Emilio Gabba, «Considerazioni sulla tradizione letteraria sulle origini della Repubblica», Les origines de la République romaine, Genève, Fondation Hardt («Entretiens sur l'Antiquité Classique», 13), 1967, p. 135-174, ici p. 157-163, sur les anticipations anachroniques de la situation italienne du $\mathrm{IV}^{\mathrm{e}}$ siècle dans ce fragment.

17. Michael H. Crawford, «From Poseidonia to Paestum via the Lucanians », Greek and Roman Colonization. Origins, Ideologies and Interactions, dir. Guy Bradley, John-Paul Wilson, Oakville, Classical Press of Wales, 2006, p. 59-72, ici p. 60.

18. A. Fraschetti, «Aristosseno », p. 101 ; p. 104 ; p. 107. 
relations de plus en plus étroites avec eux ${ }^{19}$. Les " Poséidoniates» mentionnés par Aristoxène dans le fr. 124 Wehrli doivent par conséquent être identifiés aux Lucaniens installés sur le littoral tyrrhénien ${ }^{20}$ : ceux-ci, dénonce-t-il, occupent une cité qui avait été grecque à l'origine, mais «se barbarisent» en devenant des Étrusques ou des Romains, c'est-à-dire en adoptant un idiome, des usages et des cultes qui les « romanisent » progressivement, ou qui tendent à les rapprocher de la romanité. Or les pratiques sociales de l'aristocratie lucanienne, que l'on retrouve représentées dans la peinture funéraire paestane de la fin $\mathrm{du}_{\mathrm{IV}}^{\mathrm{e}}$ siècle, révèlent indiscutablement un processus d'«auto-romanisation» qui a précédé de plusieurs décennies la déduction de la colonie de Paestum par Rome, en 273 av. J.-C. : on voit ainsi une prise de distance par rapport à la traditionnelle exaltation du guerrier et de son retour victorieux de la guerre, et une valorisation du rôle des ancêtres et du chef de famille ainsi que du magistrat qui semblent

19. Datation de l'installation des Lucaniens à Poséidonia : Jean Bérard, La colonisation grecque de l'Italie méridionale et de la Sicile dans l'Antiquité, Paris, Presses Universitaires de France, 1957, p. 222-223; Emanuele Greco, La Grande-Grèce. Histoire et archéologie [1992], trad. Annie Schnapp, Paris, Hachette, 1996, p. 136-137 ; Alfonso Mele, «Storia di Poseidonia tra VI e v secolo a.C. », I Greci in Occidente. Poseidonia e i Lucani, dir. Marina Cipriani, Fausto Longo, Naples, Electa, 1996, p. 17-19, ici p. 18. Alliance entre les Romains et Alexandre le Molosse à Poséidonia en 332 av. J.-C. : Tite-Live, vIII, 17, 9-10 ; Justin, Histoires philippiques, XII, 2, 12 ; voir F. Zevi, « Alessandro il Molosso », p. 793-832; M. Mahé-Simon, «Alexandre le Molosse et les Romains : pax ou amicitia?», Guerre et diplomatie romaines (IVe-III siècles). Pour un réexamen des sources, dir. Emmanuèle Caire, Sylvie Pittia, Aixen-Provence, Publications de l'Université de Provence, 2006, p. 197-207. Alliance ou soumission des Lucaniens aux Romains: Tite-Live, vIII, 25, 3 ; 27, 2 et 5-11; Claudius Quadrigarius, fr. 15 Chassignet (= Aulu-Gelle, Nuits attiques, VI, 11, 7) et fr. 16 Chassignet (= Aulu-Gelle, II, 19, 7) ; Diodore, $\mathrm{xx}, 104,1$; cf. Corpus Inscriptionum Latinarum $\mathrm{I}^{2}, 7$ ( $<$ L. Cornelius Scipio Barbatus [consul 298 av. J.-C.]> subigit omne Loucanam opsidesque abdoucit) ; voir A. Mele, «Le fonti storiche», Poseidonia e i Lucani, p. 68-70 ; Olivier de Cazanove, "Itinéraires et étapes de l'avancée romaine entre Samnium, Daunie, Lucanie et Étrurie », Le Censeur et les Samnites. Sur Tite-Live, livre IX, dir. D. Briquel, Jean-Paul Thuillier, Paris, Éditions rue d'Ulm, 2001, p. 147-192, ici p. 159-176 ; Ghislaine Stouder, La diplomatie romaine : histoire et représentations (396-264 avant J.-C.), thèse de Doctorat, Université d'AixMarseille, 2011, p. 377-413.

20. Strabon, VI, 1, 1-3, distingue entre deux Lucanies, celle de l'intérieur des terres et celle du littoral ; voir G. Stouder, ibid., p. 377-378; Luca Cerchiai, «I Sanniti del Tirreno: il caso di Pontecagnano », Poseidonia e $i$ Lucani, p. 73-74. 
s'inspirer des valeurs et des modèles sociaux fournis par les aristocrates romains et campaniens contemporains ${ }^{21}$.

L'archéologie et l'épigraphie fournissent une illustration de ce qu'un Grec comme Aristoxène a pu ressentir comme une lente évolution des Poséidoniates vers la romanité, et donc comme une manifestation de leur barbarisation en cours, notamment à travers l'abandon des cultes grecs traditionnels de la cité de Poséidonia. L'ancienne agora de la cité possédait un «édifice circulaire » qui a été identifié comme l'ecclésiastérion de la cité grecque, édifié vers 480/470 av. J.-C. : pourvu de gradins concentriques, ses caractéristiques le rendent comparable aux exemples métapontin et agrigentin et «permettent de l'interpréter sans ambiguïté comme le lieu de réunion de l'assemblée civique ${ }^{22}$. L'ecclésiastérion était enfermé dans un espace sacré délimité par un mur de péribole qui intégrait également un petit temple situé à l'ouest : des situations comparables à Métaponte et à Agrigente, où une inscription et un temple dédié à Zeus Agoraios indiquaient que l'agora était un «espace placé sous la protection de Zeus », permettent de penser que le petit temple poséidoniate devait être dédié à Zeus Agoraios $^{23}$. L'ecclésiastérion de Poséidonia possédait une tribune placée sous la protection d'un dieu, puisqu'elle avait été équipée d'un petit autel. Encastrée dans l'avant-dernier gradin, une stèle en

21. Angela Pontrandolfo Greco, «Segni di trasformazioni sociali a Poseidonia tra la fine del v e gli inizi del III sec. a.C. », Dialoghi di Archeologia, t. 2, fasc. 1 (n.s.), 1979, p. 27-50; Ead., « Le necropoli dalla città greca alla colonia latina », Poseidonia - Paestum, t. II : L'agora, dir. E. Greco, Dinu Theodorescu, Rome, École française de Rome ( «Collection de l'École française de Rome », 42), 1983, p. 225-265, ici p. 240-244 ; Ead., «Trasformazioni nella società pestana dell'inoltranto IV secolo », Poseidonia e i Lucani, p. 289-292; A. Pontrandolfo Greco, Agnès Rouveret, "Le necropoli urbane e il fenomeno delle tombe dipinte », Poseidonia e i Lucani, p. 159-165 ; Ead., Le tombe dipinte di Paestum, Modène, Franco Cosimo Panini, 1992, en particulier p. 18-20, et passim ; Marina Cipriani, «Prime presenze italiche organizzate alle porte di Poseidonia », ibid., p. 119-139 ; E. Greco, « Archeologia della colonia latina di Paestum », Dialoghi di Archeologia, t. 3, fasc. 6 (n.s.), 1988, p. 79-80.

22. D. Theodorescu, «Édifice circulaire. B) Architecture et restitution», Poseidonia-Paestum, t. 2, p. 39-49, ici p. 43-44.

23. Ilaria D'Ambrosio, «La città ed il territorio nel IV secolo. Catalogo », Poseidonia e i Lucani, p. 189-191, fiche 87 (« Il tempietto T )) ; E. Greco, « L'ekklesiasterion di Poseidonia - Paestum », Rites et espaces en pays celte et méditerranéen. Étude comparée à partir du sanctuaire d'Acy-Romance, dir. Stéphane Verger, Rome, École française de Rome («Collection de l'École française de Rome », 276), 2000, p. 337-340, ici p. 339. 
calcaire gris datant de la fin du $\mathrm{IV}^{\mathrm{e}}$ ou du tout début du $\mathrm{III}^{\mathrm{e}}$ siècle porte, sur quatre lignes, une inscription peinte en rouge et écrite en alphabet grec, mais en langue osque (fig. 1$)^{24}$ :

\begin{tabular}{|c|c|}
\hline Texte original : & Traduction latine : \\
\hline$\Sigma] \tau \alpha[\tau] \iota \sigma[\ldots] \imath$ & Statius $[\ldots] i$ \\
\hline$\varepsilon \varsigma$ Iovf $\eta 1[\ldots] \alpha$ & us Iovi a \\
\hline$v \alpha \rho \eta \iota 1 \alpha[\delta] \mathrm{f} \varepsilon \delta$ & nari dicavit (?) \\
\hline$\beta \rho \alpha \tau \eta \iota \varsigma \delta \alpha \tau \alpha \varsigma$ & gratiae datae \\
\hline
\end{tabular}

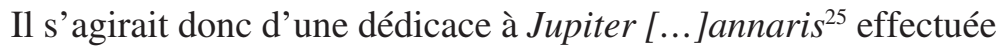
par un certain Statis [Statil]ies (?), à la suite d'une faveur <divine> reçue (brateis data). La stèle devait soutenir une statuette en bronze du dieu, dans lequel il faut sans doute reconnaître l'équivalent osque du Zeus Agoraios des cités grecques. L'anthroponyme Statis [Statil]ies (?) est clairement d'origine lucanienne et doit appartenir à un membre de l'élite au pouvoir à Poséidonia à l'extrême fin du $\mathrm{IV}^{\mathrm{e}} \mathrm{ou}$ au tout début du $\mathrm{III}^{\mathrm{e}}$ siècle, vraisemblablement un magistrat (meddix) de la cité gréco-lucanienne ${ }^{26}$. L'usage de l'écriture par les Lucaniens n'est attesté qu'après leur installation dans la région où ils sont connus à l'époque historique, et l'utilisation de l'alphabet grec s'explique par le prestige du modèle culturel grec pour les élites de langue osque en contact permanent avec les cités grecques d'Italie du Sud ${ }^{27}$. Mais les caractéristiques du dialecte osque utilisé rattache celui-ci davantage à l'osque campanien, voire au latin, qu'à l'osque utilisé par les Lucaniens de l'intérieur des terres : la

24. Loretta Del Tutto Palma, Le iscrizioni della Lucania Preromana, Padoue, Dipartimento di Linguistica, 1990, p. 52-56 ; E. Greco, « Édifice circulaire. C) La stele con iscrizione osca », Poseidonia - Paestum, t. 2, p. 137-138 et fig. 72 ; Helmut Rix, Sabellische Texte, Heidelberg, Winter, 2002 (Lu 14). Traduction latine proposée par E. Greco, loc. cit.

25. L'épiclèse du dieu est difficile à restituer; le datif Iovf construit avec une diphtongue initiale [iuvei] qui rapproche l'osque parlé par les Lucaniens de Poséidonia de l'osque parlé par les Campaniens, proche de la forme latine Iovi, tandis que dans le sanctuaire lucanien de Rossano di Vaglio (en Lucanie « intérieure ») est attestée la forme plus ancienne et plus conservatrice $\Delta 1 \omega F \eta 1 \varsigma$ [Dioueis], qui sonorise encore l'initiale di, comme on le trouve également dans la table osque de Bantia : Mauro Cristofani, «La scrittura e la lingua », Poseidonia e i Lucani, p. 201-203.

26. I. D'Ambrosio, «Topografia e urbanistica : dalla fondazione alla conquista lucana. Catalogo », ibid., p. 27-28, fiche 2 («Ekklesiasterion »).

27. M. Cristofani, «La scrittura », p. 201. 
linguistique semble confirmer la distinction que faisait Strabon entre les deux Lucanies $^{28}$, et montre l'attraction culturelle et sociale exercée par l'aire romano-campanienne sur les élites lucaniennes de Poséidonia à la fin du $\mathrm{IV}^{\mathrm{e}}$ siècle. Si l'interprétation de certains numismates est exacte, les premières monnaies de bronze avec la légende PAISTANO dateraient de la fin du $\mathrm{IV}^{\mathrm{e}}$ siècle ou du début $\mathrm{du}$ III ${ }^{\mathrm{e}}$, ce qui signifierait l'abandon par les élites lucaniennes du nom grec de la cité, remplacé par un nom indigène d'origine osque avant même la déduction de la colonie latine par les Romains ${ }^{29}$. Aristoxène ne semble donc pas avoir eu raison au sujet de l'abandon des cultes et des fêtes religieuses d'origine grecque, puisque ceux-ci étaient apparemment poursuivis et transposés en langue osque à l'époque lucanienne, mais l'abandon du grec au profit d'un dialecte italique proche du latin pour certaines cérémonies officielles, notamment religieuses, pourrait bien, en définitive, être apparu à ses yeux comme un signe manifeste de la «barbarisation » des habitants de Poséidonia, surtout si, de surcroît, l'ancienne cité grecque a fini par prendre, après la conquête lucanienne et dès la fin du IV ${ }^{\mathrm{e}}$ siècle, le nom osque de Paistom $^{30}$.

28. Voir supra n. 20 et n. 25.

29. A. Pontrandolfo Greco, «Per una puntualizzazione della cronologia delle

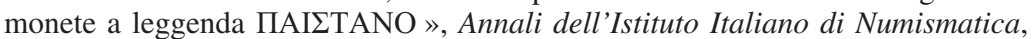
t. 30, 1983, p. 63-81; Giovanni Pugliese Carratelli, «Problemi della storia di Paestum », La monetazione di bronzo di Poseidonia-Paestum, p. 3-10; Id., «Per la storia di Poseidonia », Poseidonia-Paestum, t. 2, p. 13-31, ici p. 21 et p. 30 ; A. Mele, «Le fonti storiche», p. 70 ; Marina Taliercio Mensitieri, «La monetazione di Poseidonia-Paestum dall'occupazione lucana alla colonia latina », Poseidonia e i Lucani, p. 210-214, ici p. 211-212; contra: M. H. Crawford, «From Poseidonia to Paestum », p. 64.

30. De manière un peu ambiguë, Strabon fait un rappel historique de « la ville de Poséidonia Paistos » en évoquant successivement la présence des Sybarites, des Lucaniens et des Romains (Géographie, V, 4, 13). 


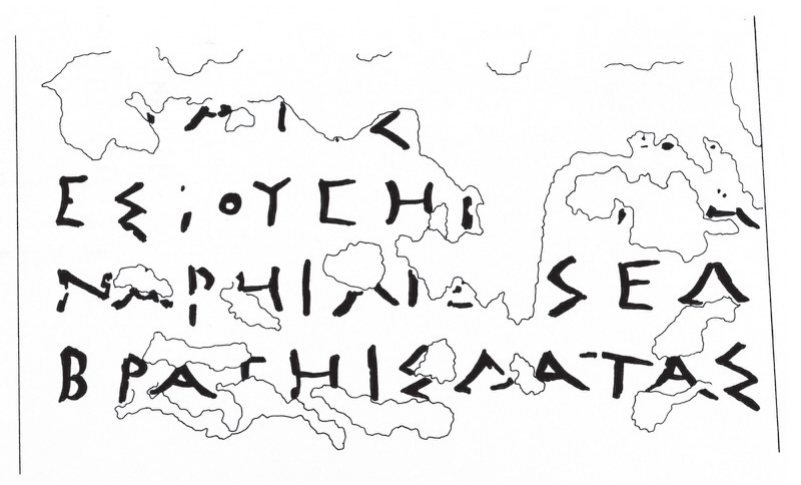

Figure 1 : L'inscription osque de la stèle de l'ecclésiastérion (E. Greco, D. Theodorescu, Poseidonia - Paestum, t. II : L'agora, Rome, École française de Rome [CEFR 42], fig. 72)

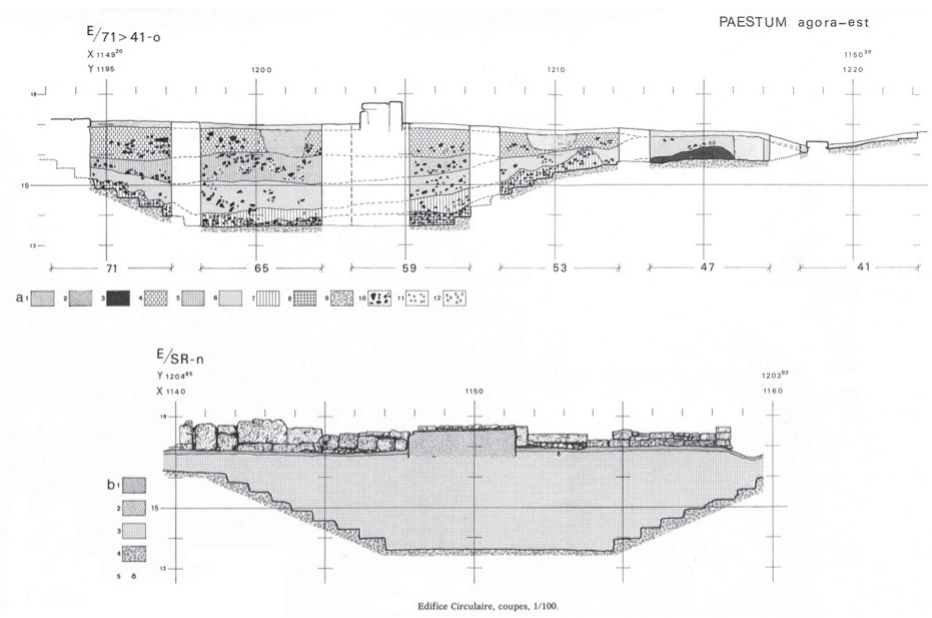

Figure 2: L'ecclésiastérion de l'agora: coupe et stratigraphie (E. Greco, D. Theodorescu, Poseidonia - Paestum, t. II : L'agora, Rome, École française de Rome [CEFR 42], fig. 29) 


\section{La déduction coloniale de Paestum et la fin des Cultes DE LA CITÉ GRÉCO-LUCANIENNE}

Aussitôt après la déduction de la colonie latine par Rome en 273 av. J.-C., l'ancienne agora grecque a été abandonnée et l'ancien ecclésiastérion fut détruit de fond en comble. Simultanément, un nouveau forum fut créé au sud de l'ancienne agora et fut équipé d'un ensemble monumental curia-comitium conforme au modèle romain ${ }^{31}$. L'archéologie témoigne de la destruction volontaire et délibérée de l'ecclésiastérion de l'agora grecque et de l'existence de rites religieux qui semblent avoir accompagné cette destruction. Le matériel céramique ainsi que quelques pièces de monnaie retrouvées dans le remblai avec lequel l'ecclésiastérion fut rebouché permettent de dater l'opération de destruction autour de 270 av. J.-C., donc immédiatement après la déduction de la colonie latine de Paestum ${ }^{32}$ (fig. 2). L'ancien ecclésiastérion de la cité grecque fut dépouillé de son revêtement en blocs de travertin, le mur de péribole qui l'entourait fut démonté, la statuette en bronze qui surmontait la stèle épigraphique fut enlevée et l'édifice circulaire fut entièrement enterré. À l'emplacement de l'édifice circulaire, et se surimposant à lui, un « complexe sacral » a été installé à l'époque romaine ; il a été équipé d'un enclos défini comme un «sanctuaire romain » et contenant deux pièces aménagées dans l'angle sud-est (un sacellum ?), d'une fontaine qui approvisionnait le sanctuaire en eau et d'un petit édifice au sud-est, transformé en maison d'habitation à l'époque impériale ( ${ }^{\mathrm{er}}-\mathrm{II}^{\mathrm{e}}$ siècles apr. J.-C.). Pour D. Theodorescu, «l'impression qui se dégage de l'ensemble est celle d'une zone réservée, dès le début de la colonie, à des édifices à caractère sacré où, même à une époque d'extension maximale de l'établissement romain, les programmes domestiques n'ont pas été acceptés ${ }^{33}$. La déduction de la colonie par Rome s'est donc traduite par une défonctionnalisation et une destruction complète de l'ancienne agora et de son monument le plus emblématique, le

31. D. Theodorescu, « Topographie et urbanisme », dans Poseidonia-Paestum, t. 2, p. 63-71, ici p. 70-71; E. Greco, "Qualche riflessione sulla storia dei monumenti », ibid., p. 72-84, ici p. 83-84 ; M. Torelli, Tota Italia, p. 46-49.

32. E. Greco, «L'édifice circulaire. A) Lo scavo », Poseidonia - Paestum, t. 2, p. 34-39, ici p. 38 ; contra : M. H. Crawford, «From Poseidonia to Paestum », p. 65-66 (édifice utilisé jusque vers 200 av. J.-C.).

33. D. Theodorescu, «Édifice circulaire. B)», p. 49. 
lieu de réunion de l'assemblée du peuple (l'ecclésiastérion), tout en préservant le caractère sacré de cet espace.

Bien plus: dans les couches les plus profondes du remblai qui servit à combler l'ecclésiastérion ont été retrouvés les ossements d'une cinquantaine de bovins adultes (fig. 2). Selon M. Leguilloux, l'excellent état de conservation des ossements de ces bovins «atteste un enfouissement très rapide après l'abattage et le dépeçage des carcasses $»^{34}$. Ces animaux semblent avoir été abattus au même moment, selon des procédés de mise à mort qui évoquent un sacrifice: "la présence de la plupart des ossements du squelette serait plutôt un indice en faveur de l'origine romaine des sacrificateurs, ce que semble confirmer par ailleurs le mode de distribution de la viande $»^{35}$. Il pourrait s'agir en fait d'une véritable «hécatombe», même si une cinquantaine d'individus ne correspondent pas exactement à ce type de sacrifice, car on sait aujourd'hui que «ce terme était couramment utilisé » et que «le nombre de cent animaux n'était pas toujours respecté, <car> il s'agissait parfois d'un nombre supérieur, mais le plus souvent d'un nombre inférieur $\star^{36}$. Dès 1983, E. Greco formulait l'hypothèse que ces nombreux ossements de bovins pourraient provenir d'un sacrifice de «purification » du site, surtout si on considère qu'un sanctuaire fut édifié par-dessus l'édifice circulaire : selon lui, il aurait pu s'agir du sacrifice qui aurait été accompli pour « inaugurer»le sanctuaire romain qui fut érigé à l'emplacement de l'ancien édifice d'assemblée $\operatorname{grec}^{37}$. Mais l'hypothèse retenue aujourd'hui est qu'il s'agirait d'un sacrifice expiatoire destiné au Zeus/Jupiter de l'ecclésiastérion lors du comblement de l'édifice circulaire par les colons romains, au moment où ceux-ci transférèrent les activités civiques de l'ancienne agora au nouveau forum de la colonie, lorsqu'ils inaugurèrent l'ensemble curia-comitium qui remplaça le bouleutérion et l'ecclésiastérion de la cité gréco-lucanienne ${ }^{38}$. Autrement dit, on aurait là la trace matérielle de l'arrêt brutal du

34. Martine Leguilloux, «L'hécatombe de l'ekklesiasterion de Poseidonia/ Paestum. Le témoignage de la faune », Rites et espaces, p. 341-351, ici p. 343.

35. Ibid., p. 347.

36. Ibid., p. 343-344.

37. E. Greco, «L'édifice circulaire. A)», p. 38.

38. E. Greco, «L'ekklesiasterion», p. 337-340; M. Leguilloux, «L'hécatombe », p. 351. 
culte civique rendu au dieu sous la protection duquel était placée l'ancienne agora gréco-lucanienne : la fin de ce culte correspondrait ainsi à la mise en place des institutions de la colonie déduite sur place par Rome en 273 av. J.-C.

L'ampleur exceptionnelle de ce sacrifice, par le coût matériel qu'il a dû représenter et par la quantité de viande qui a dû être consommée simultanément, ne peut s'expliquer que s'il a concerné et impliqué une grande partie de la population de la cité de Paestum, et que s'il représentait un enjeu politique et symbolique majeur pour l'ensemble de la colonie. Or l'ensemble des informations archéologiques sur les débuts de la colonie montre une très étroite intégration sociale entre les colons venus de Rome (environ 3000 personnes) et la population gréco-lucanienne présente sur place antérieurement ${ }^{39}$. La colonie déduite à Paestum en 273 av. J.-C. était de «droit latin», ce qui non seulement lui apportait un certain nombre de garanties juridiques et de privilèges dans ses relations avec Rome, mais déterminait également la composition de la population civique de la nouvelle «cité » qui était ainsi fondée ${ }^{40}$. Celle-ci était en effet composée, pour une partie, d'anciens citoyens romains qui acceptaient de perdre la citoyenneté romaine en échange de la nouvelle citoyenneté que leur offrait la colonie et, pour une autre partie, d'anciens habitants indigènes, donc des Grecs et des Lucaniens qui pouvaient conserver une partie de leurs terres ou de leurs privilèges sociaux en échange de cette nouvelle citoyenneté. Même s'il semble y avoir eu, au sein de la population de la colonie, une forte empreinte politique et sociale de la plèbe urbaine romaine, la société indigène gréco-lucanienne n'avait pas pour autant disparu, bien au contraire : l'onomastique des magistrats de la colonie qui sont connus par l'épigraphie pour la période républicaine montre le maintien des noms osques qui ont été latinisés (Ceppius, Digitius, Egnius, Mineius, Statius, Venneius [ou Bennius], Vibius, etc.), ce qui indique l'implication de l'ancienne élite lucanienne dans la direction de la nouvelle

39. E. Greco, «Qualche riflessione », p. 83-84 ; M. Torelli, «Paestum romana », p. 39-41; Id., Tota Italia, p. 45-46 ; D. Theodorescu, «Éléments d'urbanisme et de topographie. État actuel et perspectives », Poseidonia - Paestum, t. 2, p. 501-540, en particulier p. 526-538.

40. David Kremer, Ius Latinum. Le concept de droit latin sous la République et l'Empire, Paris, De Boccard, 2005, en particulier p. 41-110. 
cité, aux côtés de Rome ${ }^{41}$. L'ampleur sans précédent du sacrifice collectif organisé dans l'ancien ecclésiastérion avant sa destruction et son remblaiement peut donc s'expliquer par la mobilisation des anciens habitants d'origine gréco-lucanienne aux côtés des nouveaux habitants d'origine romaine, pour constituer le nouveau corps civique de la colonie «latine » de Paestum.

Au moment de la déduction de la colonie, même si une cité antérieure était préexistante (comme à Poséidonia-Paestum), la nouvelle cité était fondée sur le modèle de la fondation de Rome dont elle devenait symboliquement une «reproduction» (simulacrum) et une «image en miniature»(parva effigies $)^{42}$. Le responsable de la déduction de la colonie, membre de la commission triumvirale désignée par le sénat romain et véritable «petit Romulus » local, suivait le modèle «romuléen» de la fondation d'une citét3 : prise d'auspices inaugurale, creusement du sillon primordial pour délimiter le pomerium et offrandes de fondation, constituées des restes du sacrifice inaugural, des prémices des récoltes et peut-être aussi de divers éléments végétaux ou minéraux apportés par les colons depuis leurs territoires d'origine respectifs ${ }^{44}$. D'après Plutarque, qui s'inspire

41. M. Mello, Paestum Romana. Ricerche storiche, Rome, Istituto italiano per la storia antica, 1974, p. 13-108 et p. 110-112 ; Flaminia Arcuri, «In margine ad alcune epigrafi romane di Paestum », Bollettino storico di Salerno e Principato Citra, t. 4, 1986, p. 5-15 ; M. Torelli, «Paestum romana», p. 93-95 ; Id., Tota Italia, p. 76-77.

42. Aulu-Gelle, Nuits attiques, xvI, 13, 9. Comme l'a finement remarqué P. Zanker, c'est de la «grandeur» (amplitudo) et de la «majesté » (maiestas) du peuple romain que les colonies fondées par Rome auraient été, selon Aulu-Gelle, des « images en réduction et des reproductions », et non pas de l'apparence de la ville (urbs) elle-même : "The city as symbol : Rome and the creation of an urban image », Romanization and the City. Creation, Transformations, and Failures. Proceedings of a conference held at the American Academy in Rome to celebrate the 50th anniversary of the excavations at Cosa (14-16 May, 1998), dir. Elizabeth Fentress, Journal of Roman Archaeology, t. 38 (Suppl.), 2000, p. 25-41.

43. Jean-Michel David, «Les fondateurs et les cités », Gli statuti municipali, dir. Luigi Capogrosso Colognesi, Emilio Gabba, Pavie, IUSS Press, 2006, p. 723741, ici p. 738.

44. Caton, Origines, fr. I, 18 Chassignet $=18$ Peter (chez Servius, Commentaire sur l'Énéide de Virgile, v, 755) ; Varron, La langue latine, v, 143 ; L'agriculture, II, 1, 9-10 ; Denys d'Halicarnasse, Antiquités romaines, I, 88, 1-2 ; Ovide, Fastes, IV, 820-825 ; Plutarque, Romulus, 11, 1-5 ; Festus, p. 358 Lindsay, s.v. «Rituales libri »; Aulu-Gelle, Nuits attiques, XIII, 14, 1-3 ; Macrobe, Saturnales, v, 19, 13. Voir M. Humm, «La fondation de colonies par Rome à l'époque médiorépublicaine et le modèle de la "cité idéale" » (à paraître). 
probablement de Varron, ces offrandes de fondation étaient déposées dans une fosse de fondation qu'il appelle mundus ${ }^{45}$ : cette identification de la fosse de fondation avec le mundus est probablement une erreur ou une confusion de la source suivie par Plutarque, puisque le mundus était, à Rome, un sanctuaire souterrain situé à côté du Comitium et dans lequel il était possible de descendre pour rendre des sacrifices à certains moments de l'année ${ }^{46}$. Par contre, dans le cas de Paestum, le sacrifice collectif d'une cinquantaine de bovins, dont les squelettes ont été déposés au fond de l'ancien ecclésiastérion avant d'être recouverts par un remblai de terre, n'est pas sans évoquer le rituel décrit par Plutarque et pourrait correspondre aux pratiques rituelles liées à la fondation de la nouvelle cité. Les rites de fondation qui ont été suivis lors de la déduction de la colonie de Paestum semblent en effet avoir été en tous points conformes à ceux qui ont pu être identifiés pour d'autres colonies déduites par Rome à l'époque médio-républicaine, notamment à Cosa, la colonie jumelle fondée la même année ${ }^{47}$.

Le gigantesque sacrifice collectif d'une cinquantaine de bovins, dans la période qui suivit immédiatement la déduction de la colonie de Paestum, ne serait donc pas tant un sacrifice expiatoire que le sacrifice lié aux rituels de fondation de la nouvelle cité. En même temps, si ces rites de fondation ont eu lieu à l'emplacement du monument qui symbolisait le cœur politique de l'ancienne

45. Plutarque, Vie de Romulus, 11, 2 ; Varron, La langue latine, v, 143 ; voir le commentaire de Carmine Ampolo, dans Plutarco, Le vite di Teseo e di Romolo [1988], dir. Carmine Ampolo, Mario Manfredini, Milan, Fondazione Lorenzo Valla - Arnoldo Mondadori, 1993, p. 298-300.

46. Filippo Coarelli, s.v. "Mundus », dans Lexicon Topographicum Urbis Romae, III (H-O), dir. Eva Margareta Steinby, Rome, Quasar, 1996, p. 288-289 ; M. Humm, «Le mundus et le Comitium : représentations symboliques de l'espace de la cité », Histoire urbaine, 10, Pouvoirs, violences et sécurité dans l'espace urbain: ruptures et continuité de l'Antiquité au haut Moyen Âge, dir. Michèle Gaillard, M. Humm, 2004, p. 43-61.

47. Frank Edward Brown, Emeline Hill Richardson, Lawrence Richardson Jr., Cosa II. The Temples of the Arx, Rome, American Academy in Rome (MAAR 26), 1960, p. 9-16 ; F. E. Brown, Cosa. The Making of a Roman Town, Ann Arbor, University of Michigan Press, 1980, p. 16-17; d'autres dépôts de fondation semblent avoir été retrouvés à Artena (Roger Lambrechts, Artena 3. Un «mundus » sur le Piano della Cività, Bruxelles, Rome, Institut historique belge de Rome, 1996) et à Norba (M. Torelli, «L'età regia e repubblicana », Storia dell'urbanistica. Il mondo romano, dir. P. Gros, M. Torelli, Bari, Rome, Laterza, p. 3-164, en part. p. 134). 
cité de Poséidonia, juste avant sa destruction définitive et son remplacement par l'ensemble curia-comitium sur le nouveau forum de la colonie, cette « hécatombe », à laquelle ont pu participer aussi bien les colons (coloni) venus de Rome que les habitants (incolae) qui venaient de l'ancienne cité gréco-lucanienne, marquait à la fois la fin des anciens cultes de Poséidonia et le début des cultes de la nouvelle cité de Paestum. Et le maintien, jusqu'en pleine période impériale, d'un espace sacré à l'emplacement de l'ancienne agora suggère que celui-ci n'était pas uniquement lié au souvenir religieux fossilisé de l'ancienne cité gréco-lucanienne, mais qu'il se rattachait aussi aux fondements religieux et politiques de la colonie.

\section{Conclusion}

Finalement, on a eu à la fois tort et raison d'associer le témoignage d'Aristoxène sur la «barbarisation » des Poséidoniates avec la déduction de la colonie de Paestum par les Romains en 273 av. J.-C. ${ }^{48}$. Historiquement et chronologiquement, le philosophe n'a pas pu avoir connaissance de la déduction de la colonie, mais le phénomène culturel qu'il décrit, la désaffection de la langue grecque, notamment dans les cultes publics de la cité, était un processus largement en cours à la fin du IV siècle. L'attraction politique et culturelle que Rome exerçait sur une partie des élites aristocratiques lucaniennes pouvait largement être ressentie, par un Grec d'Italie fervent partisan de l'hellénisme tarentin en même temps que disciple d'Aristote, comme le signe d'une «barbarisation» qui finirait tôt ou tard par se traduire, après l'abandon de la langue et du mode de vie des Grecs, par l'abandon des cultes qui fondaient l'essence de l'hellénisme. $\mathrm{Si}$ ce processus d'acculturation était en cours à Poséidonia tout au long de la seconde moitié du $\mathrm{IV}^{\mathrm{e}}$ siècle, et fut favorisé par l'avancée diplomatique et militaire des Romains en Campanie et en Italie du Sud, il s'inscrivait dans la durée et le temps long, mais se conclut brutalement en 273 av. J.-C. par la déduction de la colonie de Paestum et la fondation sur place d'une nouvelle

48. Voir supra n. 5. 
cité. Contrairement à Cosa, où la déduction fut réalisée dans un espace quasi vierge ne nécessitant pas la prise en compte de la situation religieuse antérieure, la fondation d'une nouvelle cité à Paestum, qui se superposait à l'ancienne cité gréco-lucanienne de Poséidonia, nécessita de mettre brutalement fin aux cultes les plus emblématiques de l'ancienne communauté civique, en même temps qu'étaient établis ceux de la nouvelle citée ${ }^{49}$. Pire que ne l'avait imaginé Aristoxène, les anciens habitants de la cité n'auront même plus l'occasion de se réunir une fois l'an dans une fête religieuse pour parler le grec et se souvenir, en se lamentant, de leurs anciennes institutions: les dieux de la cité grecque de Poséidonia étaient définitivement morts et avaient été remplacés par les dieux romains de la colonie de Paestum.

michel.humm@unistra.fr

49. Sur l'organisation cultuelle des colonies comme reflet des relations entre colons et indigènes et sur les lieux de culte comme possibles instruments, parmi d'autres, d'une gestion pacifiée de l'après-guerre, voir Audrey Bertrand, La religion publique des colonies dans l'Italie républicaine et impériale (Italie médio-adriatique, III ${ }^{e}$ s. av. n.è-II ${ }^{e}$ s. de n. è), Rome, École française de Rome (« Bibliothèque des Écoles Françaises d'Athènes et de Rome », 365), 2016, p. 173 ; mais la volonté d'intégrer les élites indigènes par la reprise des cultes et des lieux de culte préexistants n'a pas empêché que des modifications parfois «brutales » aient été introduites dans le paysage religieux de la colonie de Paestum : voir M. Torelli, «Paestum Romana », p. 83-86 (à propos de l'installation du culte de Mens sur le forum, ou de la transformation du sanctuaire d'Héra en temple de Junon Regina ou de Junon Moneta Regina). 